Special reference is made in the report to the investigations of the Department of Research in Pure Chemistry on the synthesis of new anti-malarials, particularly the application of the novel hydroxyethylating agents, and a hydroxyethyl analogue of 'Pamaquin' has shown considerable promise. Sulphur derivatives of quinoline have also been prepared, as well as ten new bases of 2-styrylquinoline type in which a modification of Brahmachari's method raised the yield from $2 \cdot 5$ per cent to 80 per cent. Conditions have also been established for the formation of the $p$-dialkylaminobenzylidene diquinaldines, and four new 4-p-dialkylaminostyryl-quinolines have also been prepared as well as 6-hydroxyethoxylepidine and the corresponding 6-hydroxy derivatives. The pharmacological properties of the hydroxyethyl-ether derivative of morphine have been determined, and an intensive search made for morphine derivatives free from the medically undesirable side-effects and less liable to cause addiction. The Institute is also participating in the thirteenth revision of the Pharmacopeia of the United States, which is now under way.

Studies of the physical and chemical properties of synthetic racemic menthol have been published, and a method developed for determining iodine in organic compounds. The Institute is also cooperating in an important investigation of chemical methods of analysis for the highly insoluble glass required for ampoules for penicillin and organic arsenicals.

\section{A RADIO NAVIGATIONAL AID}

A NOVEL method of position-finding by the aid of long radio waves, applicable to both aerial and marine navigation, has been developed to practical utility during the War by the Decca Record Co., Ltd.

The system uses two or more fixed transmitting stations which radiate signals of constant frequency and amplitude in all directions along the ground. At the receiving point the difference in phase of the waves arriving from two stations is measured, and it is then known that the receiver is on one of a number of hyperbolic curves, which are the loci of points of equal phase difference from the two transmitters. By repeating the observation on the signals from a third station compared with one of the first two, the position on an intersecting series of curves is found, and so the exact location of the receiver is determined. The phase comparison must be carried out on signals of identical frequency, but since only signals of different frequency can be received separately, the transmitting stations operate on different frequencies having a common harmonic relationship, and the necessary restoration to a common frequency for measurement purposes is carried out in the receiver itself.

In the development of the Decca Navigator system, great care has been taken to secure adequate frequency control of the transmitters and also of the reference oscillator in the receiver, which has been designed around radio circuits of high stability. The actual measurement of phase is carried out with a special meter which, by means of a train of gears and suitable indicators, displays a direct reading of the phase difference for transfer to the chart supplied for use with the instrument. Various precautions have been taken to render the whole system free from variations of phase of the signals due to instrumental changes, and the sites of the transmitting stations are selected so as to avoid so far as possible any effect of varying ground conditions on the prop. agation of the radio waves used. It is claimed that at a range of 300 miles, the navigating system is accurate to about 200 yards, and for shorter ranges it is more accurate still. Much greater ranges are possible for daylight working, but during the night the effect of ionospheric waves superimposed upon the ground waves may limit the range at which the highest accuracy can be obtained.

During the War the system was used by the Admiralty; and it has now been disclosed that it was used from $D$-day onwards to guide the leading minesweepers and landing flotillas. The system is likely to have peace-time applications to both air and marine navigation.

\section{EXCHANGES OF INORGANIC IONS THROUGH LIVING MEMBRANES}

$\mathrm{T}$

HE large differences in concentration of single ions (especially potassium and sodium) between living cells and their surroundings were discussed at the Croonian Lecture of the Royal Society*. These differences are generally maintained by transport work through the surface layer making good the simultaneous diffusion losses.

Membranes differ greatly in permeability as shown by selected examples. The blood vessels of the vertebrate central nervous system in particular show characteristics of permeability approaching those of the surfaces of many cells. They are highly permeable to water and lipoid-soluble substances, but only slightly permeable to crystalloids including ions.

A number of examples were given demonstrating the active transport of ions through membranes composed of cells (plant roots, gills of aquatic animals, intestinal epithelium, etc.) and into (or out of) individual cells (giant plant cells, root cells, erythrocytes, striated muscles, etc.). All the cells studied are freely permeable to water, but the difference between mechanically supported plant cells and naked animal protoplasts was emphasized. The latter must always be in osmotic equilibrium with their surroundings, while the former can build up a surplus pressure by active absorption of ions.

In the case of plant roots, the energy expended in absorbing anions is considerable; but in animal cells the permeability is so slight that the energy exchanges involved in active transport cannot be measured. In several cases the process depends upon the access to glucose or oxygen or both.

In cases where an active transport takes place, the passive permeability can only be measured by means of isotopes and only when special conditions are maintained. Several experiments with isotopes have been recalculated to give absolute permeability figures, and these show for erythrocytes and muscles higher values for sodium than for potassium, a result incompatible with a eimple pore permeability, but supporting the Lundegårdh conception of mosaic surface films of the Langmuir pattern.

* Abstract of the Croonian Lecture "The Active and Passive Exchanges of Inorganic Ions through the Surfaces of Living Cells Krogh, For.Mem.R.S., before the Royal Society on October 25. This Lecture should have been given in 1940, but Prof. Krogh was then unable to travel to England. 